\title{
Simulation Modeling of Thermoacoustic Cooling and Refrigeration
}

\author{
Abdullah A. Alshorman \\ Al-Balqa' Applied University-Al-Huson University College, Mechanical Engineering Department, \\ P.O. Box 50 - Al-Huson 19117-Irbid - Jordan
}

Conventional refrigeration requires comprehensive power, control, and maintenance systems, all at high costs. As a result it is promising to have an alternative method, which is capable of creating cryogenic refrigeration with no moving parts, a clean energy source, and with a relatively simple design and cheap components. This could be achieved through thermoacoustic cooling techniques, which is based on the oscillation of sound waves to circulate heat between high and low heat exchangers within a well-designed thermoacoustic device.

In this study an inclusive acoustic model has been proposed, in which the main parameters have been determined to build up a matrix of constituted engineering-mathematical equations that form the main structure of the modelling process. This modelling has coupled with its associated thermodynamical parameters to form the appropriate thermoacoustic model, which allows for the scaling of parameters, determination of performance rate, and enables refrigeration-performance enhancement.

Our results show that the model can provide a precise margin of applications for each parameter of thermoacoustic refrigeration for different working fluids. Also, it is possible to determine the numerical ranges of efficient cooling for absorbed and released heat $\left(q_{c}, q_{H}\right)$, maximum-pressure amplitude $\left(P_{m}\right)$ and sound intensity (I), respectively.

\section{INTRODUCTION}

Thermoacoustic cooling and refrigeration is an efficient method of obtaining refrigeration effects from a clean, simple, inexpensive, and compact alternative source of energy. This is the acoustic energy, which could be utilized using thermodynamics, fluid mechanics, and acoustics principles and applications.

Thermoacoustics is based on the principle that sound waves are pressure waves. These sound waves propagate through the air (or gas) via molecular collisions. The molecular collisions cause a disturbance in the air (or gas), which in turn creates constructive and destructive interferences. The constructive interference makes the molecules compress, and the destructive interference makes the molecules expand. This principle is the foundation of thermoacoustic refrigerator systems.

Fundamentally, refrigeration relies on two major thermodynamic principles. First, a fluid's temperature rises when compressed and falls when expanded. Second, when two substances are placed in direct contact, heat will flow from the hotter substance to the cooler one. While conventional refrigerators use pumps to transfer heat on a macroscopic scale, thermoacoustic refrigerators rely on sound to generate waves of pressure that alternately compress and relax the gas particles. ${ }^{1,2}$

Constructively, thermoacoustic systems do not require phase-change refrigerants and use inert gases as their working fluids, which is an environmentally benign alternative. ${ }^{3}$

In principle, thermoacoustic devices consist of resonators that contain high-amplitude standing or travelling waves. They depend on the interaction of the high-amplitude acoustic waves with surfaces in the resonator, such as a stack, to either transport heat or extract power. ${ }^{4}$ Typically, standing-wave thermoacoustic devices consist mainly of an acoustic resonator filled with a gas. In the resonator, a stack consisting of a number of parallel plates and two heat exchangers are appropriately in- stalled. In the case of an engine, the heat exchangers are used to maintain a temperature gradient over the stack. The hot-heat exchanger supplies heat $\left(Q_{H}\right)$ to the hot end of the stack, and the cold-heat exchanger extracts the heat $\left(Q_{C}\right)$ from the cold end of the stack. The stack is the heart of the engine, where the thermoacoustic cycle is generated. ${ }^{1,5}$ The stack consists of a large number of closely spaced surfaces aligned parallel to the length of the resonator tube. ${ }^{6}$ Schematic details of a thermoacoustic device including the stack are illustrated in Fig. 4.

According to the types of acoustic waves enabled by the thermoacoustic cooling systems, thermoacoustic devices can be categorised into two types: standing-wave engines and travelling-wave engines. Standing-wave engines work through thermal expansion and contraction of a gas between a hot and cold source. The result is a piston-less engine created by the unstable resonance in the system. It mainly consists of a sound-generating device, an acoustic resonator, and a stack sandwiched between an ambient-heat exchanger and a coldheat exchanger.

Thermoacoustic standing-wave and travelling-wave refrigerators are promising devices for applications/cbox, thanks to their simpl structure, high reliability, and low cost. Travellingwave coolers are highly efficient because of the reversible nature of the thermal process involved. However, very large dimensions are commonly needed for thermoacoustic machines. Whereas some practical machines, such as satellite coolers, need to be small and light-weight. ${ }^{7}$

In this study, thermodynamic and acoustical principles are analyzed and used to create a thermoacoustic model. We address the main effective parameters in this process and suggest approaches for performance enhancement of thermoacoustic devices. Moreover, the results of this modelling could be used to show a scaling visualization of the quantitative contributions of the parameters involved. 